\title{
Electron gases make their mark
}

\section{This year's Nobel Prize for Physics is awarded to Dr Klaus von Klitzing for his work on the quantum Hall effect. The development is an important pointer to a field of physics still poorly understood.}

Professor Klaus von Klitzing, director of the Max Planck institute of solid state physics at Stuttgart, said earlier this week that he hoped it would not be another 22 years before a West German won a Nobel Prize. Modestly, he was referring to the prize awarded that long ago to the Munich physicist Mossbauer for the discovery that still bears his name, and to the substantial investment in basic science by the West German government over recent decades. Most observers expect von Klitzing's hope to be fulfilled.

As with Mossbauer's work, the importance of von Klitzing's research has been quickly recognized. His first published article on what is now called the quantized Hall effect (with G. Dorda of the Siemens research laboratory at Munich and $\mathbf{M}$. Pepper of the Cavendish Laboratory, Cambridge) appeared only in 1980 (Phys. Rev. Lett. 45, 494; 1980). The objective then was to devise an accurate determination of the fine-structure constant $\left(h / e^{2}\right.$, where $h$ is Planck's constant and $e$ the electric charge of the electron) in terms of a standard of electrical resistance. As things are turning out, it seems just as likely that von Klitzing's original experiment may be used the other way around, to provide convenient and portable standards of electrical resistance that can be directly related to fundamental constants.

The essential novelty of the quantum Hall effect is that it is one of the complicated series of phenomena observed in the now fashionable "two-dimensional electron gases", the planar layers of essentially free electrons that are inferred to exist most simply at the boundaries between, say, the semiconductors GaAs and GaAlAs when these are grown together epitaxially to form a heterostructure. They also occur in an oxide layer sandwiched between a metal and a semiconductor.

These two-dimensional systems are now all the rage. The population of electrons in the transition plane can be determined at will by suitably biasing the two poles of the device. The particular interest of von Klitzing's work is that it exploits the way in which, in strong magnetic fields perpendicular to the plane of the twodimensional electron gas, the cyclotron motion of the electrons is quantized; only specified states of motion are allowed.

The analogy with the classical Hall effect is skin-deep only. That phenomenon is most simply that by which a magnetic field perpendicular to an electrical current flowing in a conductor will excite an electrical voltage in the direction at right angles to the field and to the current. Most simply, the Hall effect is a direct prediction of Maxwell's equations: alternatively, in baby-talk, it is a reflection of the tendency for electrons moving at right angles to a magnetic field to be deflected into cyclotron orbits which, being disallowed, shows up as an electrical potential across the conductor.

For practical purposes, the Hall voltage is classically proportional to the external magnetic field. The phenomenon is a convenient way of studying the properties of electrons in metals and semiconductors. It is, for example the standard way of determining electron mobility. Given the general ambition now to make a practical device of more or less any physical phenomenon, the classical Hall effect is potentially a means by which external magnetic fields might be used (without physical contacts) for generating transverse voltages in current-carrying conductors so as to make novel kinds of switches for applications not yet identified.

The quantum analogue of the classical Hall effect owes much to the work of the Soviet physicist Landau, who was the first to show that the motion of electrons constrained to move in a plane would be quantized in the presence of a perpendicular magnetic field. This leads to the conclusion that the Hall conductance (the ratio of current to transverse voltage) should itself be a multiple of the fine structure constant. (The simple expectation is that the Hall conductivity should be an integral multiple of the inverse of the finestructure constant.)

Making these phenomena susceptible to observation has been a technical triumph of the first order. Liquid helium temperatures are unavoidable. Very high magnetic fields (20 tesla or more) are usually necessary but, given the need for liquid helium anyway, may conveniently be provided by superconductors.

Von Klitzing's original measurement of the fine-structure constant in terms of a known resistance, repeated many times since 1980, has now been shown to be capable of an accuracy of a few parts in 10,000 million, although the present state of the measurements is limited by the accuracy with which the fundamental constants are known. But the availability of a system of measurement of such potential precision, greater even than that so far demonstrated for Josephson junctions, will hasten the day when it becomes prudent to fix the value of some composite constant, such as the fine-structure constant, and to allow the unit of electrical resistance to be fixed in terms only of atomic quantities such as these.

This is the promise offered when the quantum Hall effect was in its infancy. Since then, a great many surprises have made the field even more intriguing. Not the least has been the discovery that the Landau levels are not always simply quantized, but that the quantum numbers may be fractional rather than integral (or that a two-dimensional electron gas at low temperatures may behave as if it were made of particles with fractional electrical charge).

Nobody seriously suggests that physics is being turned on is head in ways like these. Rather, the inference is that the fractional quantization of the twodimensional electron gas is a measure of collective motions that are themselves quantized. In other words, the systems behave as if they were two-dimensional quantum liquids - whence the discovery that, under sufficiently extreme conditions, the liquid may be crystallized.

For the immediate future, the absorbing interest is to find a better understanding of the behaviour of these previously unknown electronic structures. The theoreticians have much to do, but the recognition that there are close similarities with at least some models of superconductivity is obviously a challenge. Meanwhile, the experimentalists are doing what they can to determine the structure of electronic states in these twodimensional structures.

Ultimately, no doubt, there will be devices as well. Perhaps the most hopeful direction in which to look for these is that the population of electrons in the twodimensional layer can so easily be altered by suitable adjustment of the electrical bias of the system, suggesting means by which conduction in the plane of the layer may be disproportionately affected. But despite the tradition that a phenomenon that does not lend itself to the construction of a laser is almost certain to make a transistor of some kind, these are early days for that train of speculation. What von Klitzing has done is to open up a whole stack of phenomena whose explanations are still incomplete. 